\title{
On the Last Geometric Statement of Jacobi
}

\author{
R. Sinclair
}

\section{CONTENTS}

1. Jacobi's Statement

2. Computing Caustics on Ellipsoids of Revolution

3. Computing Caustics on Triaxial Ellipsoids

4. Conclusion

Acknowledgments

References

2000 AMS Subject Classification: Primary 53C20; Secondary 53-04

Keywords: Conjugate locus, caustic, ellipsoid
Based upon numerical experimentation, we claim that all caustics from any nonumbilical (nonpolar for ellipsoids of revolution) point $p$ on any ellipsoid embedded in $\mathbb{R}^{3}$ (except the 2 -sphere) have exactly four cusps, all of which are on lines of curvature (meridians and parallels for ellipsoids of revolution) intersecting either $p$ (even caustics) or $-p$ (odd caustics). This is an extension of a statement usually attributed to Jacobi.

\section{JACOBI'S STATEMENT}

A basic question of differential geometry is when a given geodesic actually is a curve of shortest distance. For example, a geodesic curve $\gamma$ through a point $p$ may intersect another infinitesimally close geodesic through $p$ in another point $q . \gamma$ is the curve of shortest distance between $p$ and any point on $\gamma$ between $p$ and $q$, but it is not the curve of shortest distance between $p$ and any point on $\gamma$ after $q$. In such a case, $p$ and $q$ are said to be conjugate. See Chapter III of [Sakai 96] and also [Kobayashi $67]$ for a more detailed introduction. The locus of all points conjugate to $p$ is called the conjugate locus or (first) caustic from $p$. One can imagine that infinitesimally close geodesics will generally intersect more than once. The loci of the points of second intersection are known as the second caustic from the point $p$ and so on.

What is known as Jacobi's statement concerns the caustics from points on the surface of an ellipsoid.

Jacobi used the conjugate locus from a point on ellipsoids of revolution as an example in his sixth Lecture on Dynamics [Jacobi 84], describing it as roughly like the evolute of the ellipse (which has four cusps), and including a drawing with the four cusps labeled $A$ to $D$. In the twenty-eighth lecture of the same series, in which he integrated the geodesic flow on the triaxial ellipsoid by separation of variables, no mention was made of caustics. The result concerning integrability was and is of great importance in geometry and dynamics. See [Knörrer 80] for further historical notes and a clear description of the geometry of the geodesic flow.

(c) A K Peters, Ltd. $1058-6458 / 2003 \$ 0.50$ per page Experimental Mathematics 12:4, page 477 
A copy of an unfinished paper of Jacobi's, along with a lengthy addendum written by A. Wangerin, is included in his Collected Works [Jacobi and Wangerin 91]. This paper contains the first-order approximation (in eccentricity) to a caustic from a point on an oblate spheroid, explicitly mentioning that the earth has such a shape. The first caustic is found to be a 4-cusped hypocycloid to this order.

Von Braunmühl followed up Jacobi's work with two papers. The first, [von Braunmühl 79], treats caustics from points on various surfaces of revolution. As far as ellipsoids of revolution are concerned, there is a mostly qualitative discussion leading to the conclusion that the conjugate locus has four cusps, and some figures. He points out that these cusps appear on the meridian opposite the starting point, and the parallel of the same radius as the parallel which intersects the starting point (there are two of these, labeled $r_{0}$ and $r_{0}^{\prime}$, in Figure 2 of his paper). In a paper which also addresses issues raised in Jacobi's lectures, von Mangoldt ([von Mangoldt 81], in particular Section IV and the footnote at the bottom of page 48) provides a clarification of von Braunmühl's argument concerning the location of two of the cusps on the parallel $r_{0}^{\prime}$ just referred to.

Figure 2 of von Braunmühl's paper [von Braunmühl 79] appears again in textbooks of the following century (page 143 of [Struik 61], page 270 of [do Carmo 92], and page 296 of [Giaquinta and Hildebrandt 96], which reproduces all five figures, but with some labels removed). One of the motivations of the present work is to provide computed illustrations of caustics, in the hope that these will be both more accurate and more informative than their antique counterparts.

Von Braunmühl's second paper on this subject [von Braunmühl 82] is concerned with triaxial ellipsoids, containing explicit formulæ for the conjugate locus in terms of hyperelliptic functions (Section 9 of that paper). Figure 2 of the paper illustrates the conjugate locus as having exactly four cusps. In Section 6 of the paper, it is shown that the conjugate locus meets the two lines of curvature intersecting the antipode of the starting point (these lines of curvature are labeled $\mu_{0}^{\prime}$ and $\nu_{0}^{\prime}$ in Figure 2 of the paper) tangentially. For the definition of a line of curvature, see the remarks to Proposition 3.5.4 in [Klingenberg 82].

More recent results concerning conjugate loci from points on ellipsoids are to be found in Section 3.5 (in particular Lemma 3.5.13) of [Klingenberg 82]. Note in particular that the conjugate locus of an umbilic point on an ellipsoid is its antipodal umbilic point (Theorem
3.5.16). See also [Margerin 91], where it is shown that conjugate loci do not need to be closed. This last result makes Jacobi's statement even more remarkable.

Now let us return to the question of when a given geodesic actually is a curve of shortest distance. The cut locus from a point $p$ on a surface $S$ is the closure of the set of points that can be connected to $p$ by at least two distinct shortest paths in $S$ [Wolter 79]. On a twodimensional surface, the cut locus from a point $p$ defines a tree-shaped boundary, beyond which any geodesic curve emanating from $p$ is no longer minimizing. The concept of the cut locus was introduced under the name of "ligne de partage" in Section 2 of [Poincaré 05], where one can also find an early, clear discussion of caustics from points on convex surfaces, and their relation to the cut locus. For a modern definition of the cut locus, see Section 4 of Chapter III of [Sakai 96].

Recent experimental work [Itoh and Sinclair 02] indicates that the cut loci from points on the surfaces of triaxial ellipsoids consist of only one topological segment, and are subarcs of the line of curvature passing through the antipode of the starting point. Since the endpoints of the cut locus are conjugate points at cusps of the conjugate locus, as pointed out in [Poincaré 05], this conjecture confirms what von Braunmühl wrote (as detailed above).

M. Berger, in a historical review of Riemannian Geometry (Section TOP 4 of [Berger 00]), writes

... But this latter assumption depends on the scandalously unproved Jacobi "statement": the conjugate locus of a non-umbilical point $m$ of an ellipsoid has exactly four cusps.

Further, in a talk given by V. I. Arnold at the Fields Institute in 1997 [Arnold 99] (and see also Chapter 3 of [Arnold 94]), he says

At the end of his lecture on these caustics Jacobi remarked that for the simplest perturbation of the sphere (making it an ellipsoid) the number of cusps is $4 \ldots$ He even stated that in the case of an ellipsoid a caustic has always four cusps. I do not know whether this Jacobi statement is true or not. It is a challenge both for the algebraic geometry and for the scientific computing ... However this real problem is too difficult for the algebraic geometers ... and thus the "Last Geometric Jacobi Statement" is rather a conjecture than a theorem ... It is known that the first caustic has at least four cusps (for a convex surface). 
We have taken this statement as our starting point, and numerically investigated caustics from points on general ellipsoids.

For a review of the systematic investigation of caustics in a different context, see [Duistermaat 74, Hanyga 97]. It is also of interest to note that the caustic which is the envelope of the normals to the three-dimensional ellipsoid in $\mathbb{R}^{4}$ [Joets and Ribotta 99] has four closed curves of hyperbolic umbilics.

Caustics are of relevance to many problems in physics [Ehlers and Newman 00] and geophysics [Gjøystdal et al. 02]. They are difficult to compute, particularly in an Eulerian setting [Benamou and Solliec 00]. In particular, we want to be able to compute second and higher caustics, so we must use a Lagrangian or phase-space approach [Lambare et al. 96, Sinclair and Tanaka 02, Fomel and Sethian 02, Engquist et al. 02]. Here, we will make use of elementary differential geometry to construct algorithms specific to our needs.

\section{COMPUTING CAUSTICS ON ELLIPSOIDS OF REVOLUTION}

Let

$$
(u, v) \mapsto(r(v) \cos u, r(v) \sin u, v)
$$

be a parametrization of a class of surfaces of revolution (including all ellipsoids of revolution) embedded in $\mathbb{R}^{3}$.

Let $(u(s), v(s))$ be an arc-length parametrization of a geodesic. We wish to compute a Taylor expansion of $u$ and $v$ in terms of $s$, with $u(0)=u_{0}$ and $v(0)=v_{0}$. Since geodesics on ellipsoids of revolution generally oscillate between parallels, we introduce a further coordinate $d \in$ $\{-1,1\}$, which indicates the direction of the geodesic: $d=1$ indicates that $v$ is increasing with $s, d=-1$ that $v$ is decreasing with $s$.

Our algorithm makes use of Clairaut's Theorem (Theorem 6.4 of [Bolsinov and Fomenko 99] and Theorem 3.5.23 in [Klingenberg 82]). Recall that geodesic curves on two-dimensional surfaces of revolution are the paths of frictionless test particles moving free from external forces except the one which is necessary to constrain them to the surface. This force is (i) normal to the surface and (ii) its projection onto the plane perpendicular to the surface's axis of rotational symmetry is radial (centripetal) or null. These two properties conserve, respectively, (i) the speed and therefore also energy of the particles, and (ii) the component of angular momentum in the direction of the surface's axis of rotational symmetry. Let $c$ be Clairaut's constant, a dimensionless quantity proportional to the conserved component of angular momentum. The 4-tuple $\left(u_{0}, v_{0}, d, c\right)$ uniquely determines a geodesic curve.

Writing

$$
\begin{aligned}
r(v)= & r_{0}+r_{1}\left(v-v_{0}\right)+\frac{r_{2}}{2}\left(v-v_{0}\right)^{2} \\
& +\frac{r_{3}}{6}\left(v-v_{0}\right)^{3}+O\left(\left(v-v_{0}\right)^{4}\right),
\end{aligned}
$$

we find

$$
\begin{aligned}
v(s)= & v_{0}+\sqrt{\frac{r_{0}^{2}-c^{2}}{r_{1}^{2}+1}} \frac{d s}{r_{0}} \\
& -\frac{r_{1}\left(r_{0}^{3} r_{2}-c^{2} r_{2} r_{0}-c^{2} r_{1}^{2}-c^{2}\right) s^{2}}{2\left(r_{1}^{2}+1\right)^{2} r_{0}^{3}}+O\left(s^{3}\right) \\
u(s)= & u_{0}+\frac{c s}{r_{0}^{2}} \\
& -c \sqrt{\frac{r_{0}^{2}-c^{2}}{r_{1}^{2}+1}} \frac{r_{1} d s^{2}}{r_{0}}+O\left(s^{3}\right) .
\end{aligned}
$$

These quadratic polynomial approximations are the basis of our algorithm.

We approximate a surface of type $(2-1)$ by piecewise cubic polynomials (hence Equation (2-2)), and geodesics by piecewise quadratic polynomials. We propagate $u$ and $v$ for some small distance $\delta s$ such that the error is less than some given tolerance, then evaluate $u_{0}^{\prime}=u(\delta s)$, $v_{0}^{\prime}=v(\delta s)$ and set $d^{\prime}$ to \pm 1 depending upon the sign of $\dot{v}(\delta s) .\left(u_{0}^{\prime}, v_{0}^{\prime}, d^{\prime}, c\right)$ are then used for the next step.

The method we will use to find caustics is based upon Jacobi fields. The relationship between Jacobi fields and conjugate points is well explained in Section 7 of Chapter 27 of [Postnikov 01], and Section 2.3 of Chapter 5 of [Giaquinta and Hildebrandt 96]. We make use of one of many equivalent definitions (see Section 2 of [Kobayashi $67]$, from which we now paraphrase): The points $\gamma\left(t_{1}\right)$ and $\gamma\left(t_{2}\right)$ of a geodesic $\gamma$ are said to be conjugate if there is a geodesic variation $\gamma_{h}$ (a one-parameter family of geodesics $\gamma_{h}=\gamma_{h}(t), t_{1} \leq t \leq t_{2}$ and $-\epsilon<h<\epsilon$, such that $\gamma_{0}=\gamma$ ) which induces an infinitesimal variation vanishing at $t=t_{1}$ and $t=t_{2}$. See also the discussion of focal points in Section 4 of Chapter 10 of [do Carmo 92], and Complement 2.1.13 to Theorem 2.1.12 in [Klingenberg 82]. We apply automatic differentiation with respect to $c$ to the code which computes Equations $(2-3)$. This provides us with piecewise quadratic polynomial approximations to $d u / d c$ and $d v / d c$, as polynomials in $s$. We can compute the roots of these, and if both 


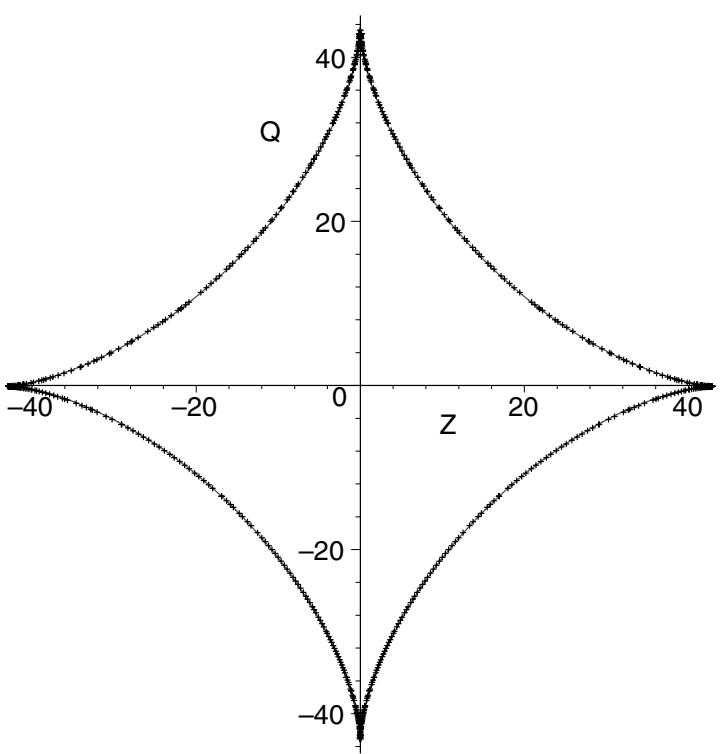

FIGURE 1. The first caustic from a point at latitude $36^{\circ} 47^{\prime}$ on the Australian National Spheroid, an oblate ellipsoid, for which the eccentricity is $e_{\text {earth }} \approx 0.08182$. All measurements are in kilometres. The crosses are numerical data. The curve is the first order approximation.

are found to go to zero at the same value (within a certain tolerance) of $s$, then that is judged to be a point of intersection of two infinitesimally close geodesics.

The algorithm need only count these, as $s$ increases, until a point on the desired caustic is found, or $s$ exceeds some maximum value.

\subsection{First-Order Approximations for Small Eccentricity}

Here we will use the geophysical motivation given in [Longuet-Higgins 90] to provide us with an example. One is interested in ascertaining the pattern of rays in the neighbourhood of the antipode of a source point on a slightly oblate spheroid (the earth), since this will give one some (clearly extremely approximate) information concerning long-range sound propagation in the ocean.

First-order approximations to the first caustic are given both in [Jacobi and Wangerin 91] and [LonguetHiggins 90]. The general shape is that of a 4-cusped hypocycloid. The earth is a good example to use since it is indeed only slightly eccentric. Using the Australian National Spheroid geometrical constants (from Table 3c of [Featherstone 96]), we have

$$
e_{\text {earth }} \approx 0.08182018 \text {. }
$$

Following Wangerin's presentation in which $\mathcal{Q}$ and $\mathcal{Z}$ are (to first order) distances from the antipode along a

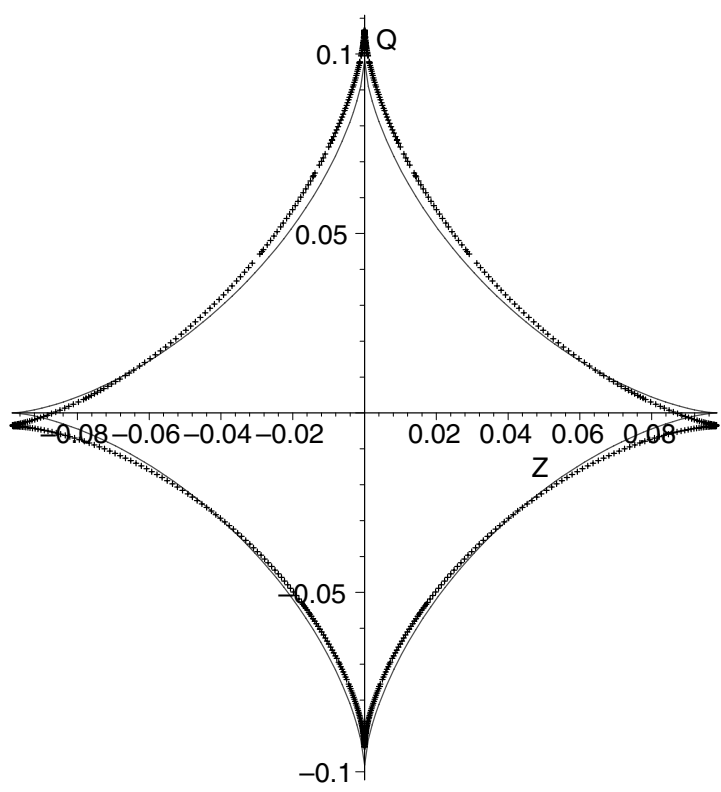

FIGURE 2. A comparison of numerical data (crosses) with the first-order approximation (solid curve) to a first caustic on an oblate spheroid of eccentricity $e_{s} \approx 0.3049$.

meridian and a parallel, respectively, we find that the first caustic from a point at latitude $36.78^{\circ}$ is given by

$$
\mathcal{Q}^{2 / 3}+\mathcal{Z}^{2 / 3}=\mathcal{C}^{2 / 3}
$$

with

$$
\mathcal{C}=42.93 \mathrm{~km} \text {. }
$$

See Figure 1. We have also checked the case of latitude $45^{\circ}$, for which $\mathcal{C} \approx 33 \mathrm{~km}$. This is in agreement with [Longuet-Higgins 90]. These results confirm the utility of the first-order approximations in naturally occurring situations, and also provide us with a first check of our software. One would also wish to have some idea of the values of eccentricity for which these approximations break down. We have therefore also investigated the ellipsoid given by

$$
\left(\frac{20}{21}\right)^{2}\left(x^{2}+y^{2}\right)+z^{2}=1,
$$

for which the eccentricity is

$$
e_{s}=\frac{\sqrt{41}}{21} \approx 0.3049
$$

using the starting point

$$
\left(\frac{21}{25}, 0, \frac{3}{5}\right) \text {. }
$$

The numerical data and first-order approximation are compared in Figure 2. Note that in this case the coordinates $\mathcal{Q}$ and $\mathcal{Z}$ are with respect to tangents to the 

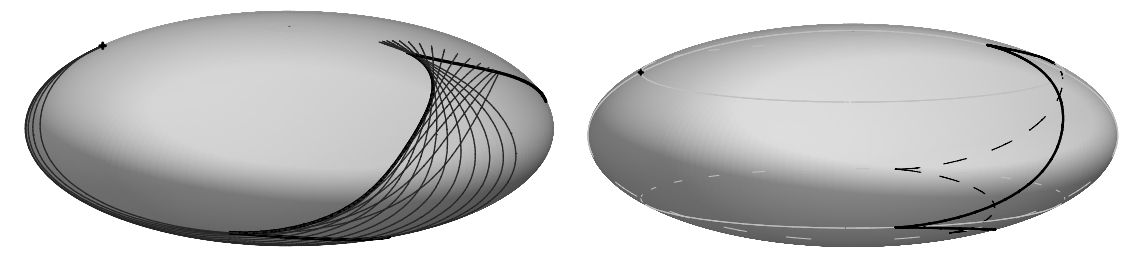

FIGURE 3. Two views of the conjugate locus (first caustic) from a point on an oblate ellipsoid, one showing the geodesics whose envelope is one arc of the caustic. The starting point is shown as a black cross.
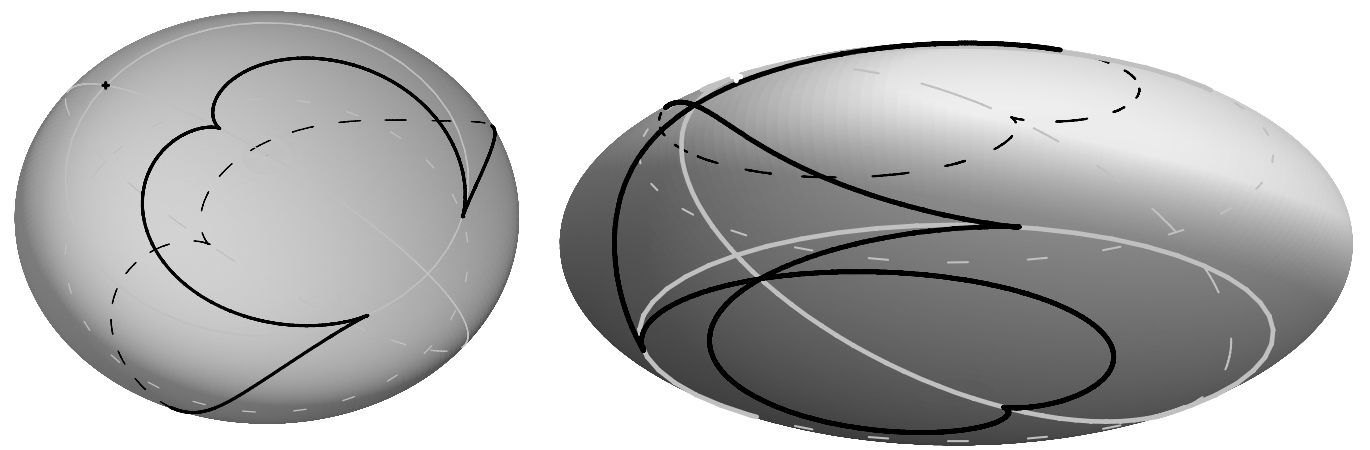

FIGURE 4. The second and third caustics from a point on an oblate ellipsoid.

meridian and the parallel passing through the antipode to the starting point, respectively. It would appear that these approximations are still qualitatively correct for such large eccentricities. What is important is that the number of cusps is still clearly four.

\subsection{Caustics on a Significantly Oblate Spheroid}

Now take the oblate spheroid (an ellipsoid of revolution) given by

$$
\left(\frac{2}{5}\right)^{2}\left(x^{2}+y^{2}\right)+z^{2}=1
$$

and the starting point

$$
\left(-2,0, \frac{3}{5}\right)
$$

The first three caustics are shown in Figures 3 and 4, all of them having four cusps. The cusps of the caustics are all on parallels or meridians intersecting the starting point or its antipode. Self-intersections of the third caustic are on the meridian intersecting the starting point.

One may be tempted to think that, particularly for even caustics, the geodesic joining the starting point to a cusp is a line of curvature. This is, however, not so, except in obvious cases. This comment applies to all ellipsoids.

\subsection{Caustics on a Prolate Spheroid}

Now take the prolate spheroid given by

$$
4\left(x^{2}+y^{2}\right)+z^{2}=1
$$

and the starting point

$$
\left(\frac{-2}{5}, 0, \frac{3}{5}\right) \text {. }
$$

The various caustics are shown in Figures 5 and 6 . The cusps of the caustics are all on parallels or meridians intersecting the starting point or its antipode. Selfintersections of the second and third caustics are on the meridian intersecting the starting point.

In all cases, the caustics have four cusps.

\section{COMPUTING CAUSTICS ON TRIAXIAL ELLIPSOIDS}

We wish to capture caustics for a small number of representative cases to an accuracy of only a few decimal places. In the spirit of fast prototyping, we have therefore chosen a robust but low-order algorithm. This algorithm will be able to treat a wide class of surfaces embedded in $\mathbb{R}^{3}$, including all ellipsoids.

We will define the surface implicitly as the set of solutions in $\mathbb{R}^{3}$ of the equation

$$
U(x, y, z)=0
$$



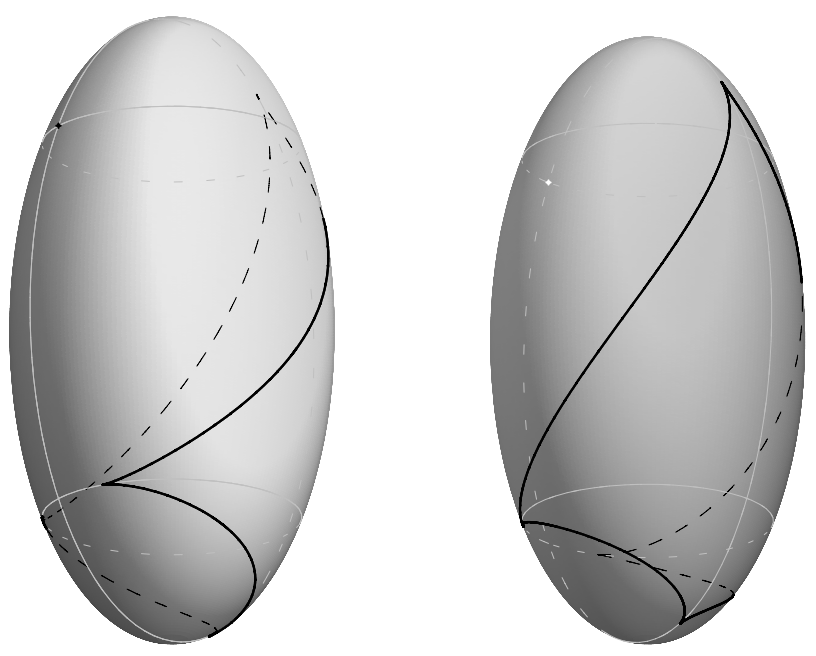

FIGURE 5. Two views of the conjugate locus (first caustic) from a point on a prolate ellipsoid. The starting point is shown as a black cross where it is in the foreground, and white where it is on the far side of the surface.
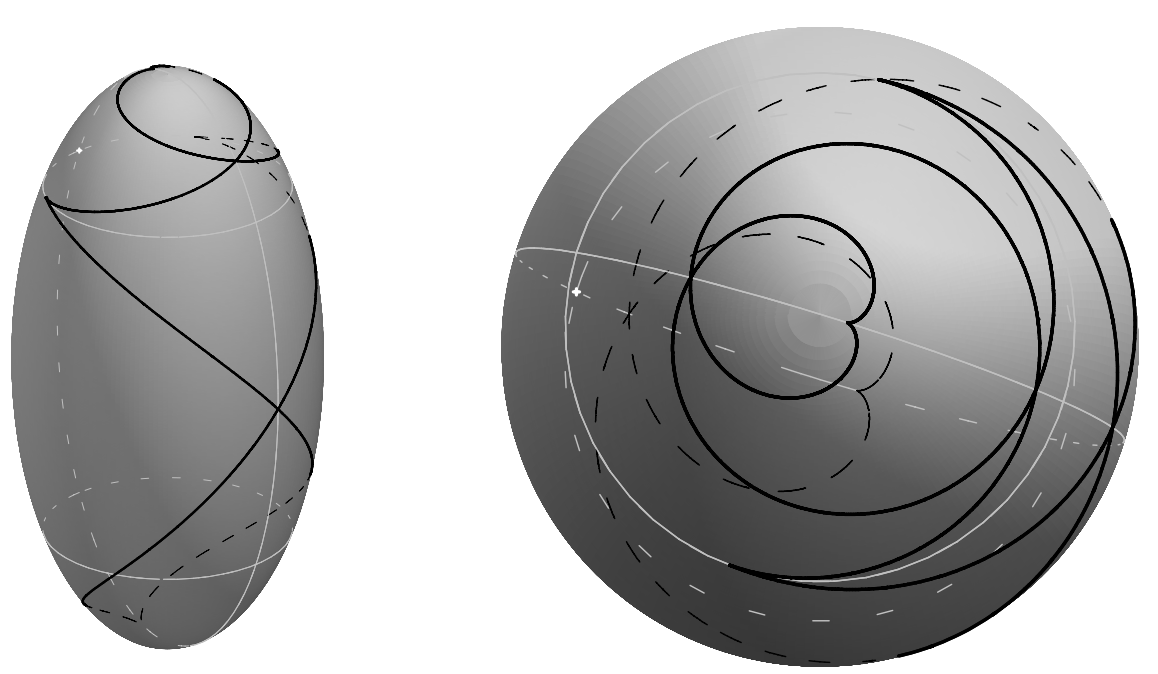

FIGURE 6. The second and third caustics from a point on a prolate ellipsoid.

where, of course, our object of interest in this paper is the family of surfaces given by

$$
U(x, y, z)=\frac{x^{2}}{a^{2}}+\frac{y^{2}}{b^{2}}+\frac{z^{2}}{c^{2}}-1 .
$$

Given a starting point $\vec{x}_{0}$ on the surface, a starting direction $\vec{v}_{0}$ tangential to the surface, and a step length $\ell$, we can compute a new position

$$
\vec{w}_{0}=\vec{x}_{0}+\ell \frac{\vec{v}_{0}}{\left\|\vec{v}_{0}\right\|}
$$

and then (approximately) project it back on to the surface using what is essentially one Newton step:

$$
\vec{x}_{1}=\vec{w}_{0}-U\left(\vec{w}_{0}\right) \frac{\nabla U\left(\vec{w}_{0}\right)}{\left\|\nabla U\left(\vec{w}_{0}\right)\right\|^{2}} .
$$




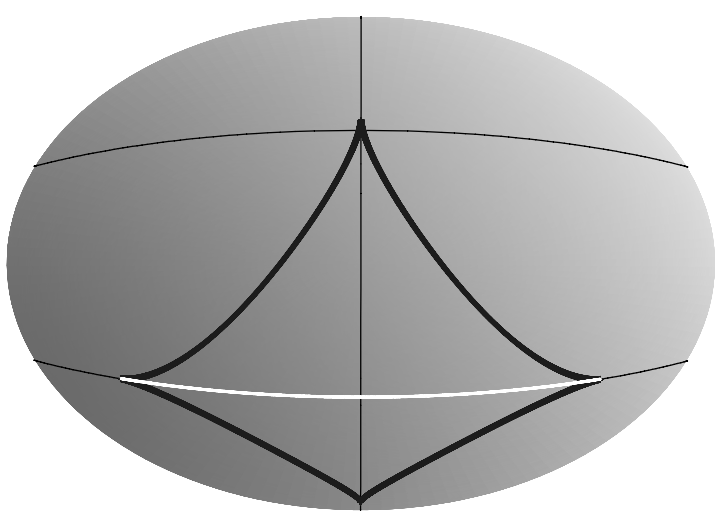

FIGURE 7. Frontal view of the conjugate locus (in black) from a symmetric point on a triaxial ellipsoid. The white curve is the cut locus from the same point. The thin black curves are lines of curvature.
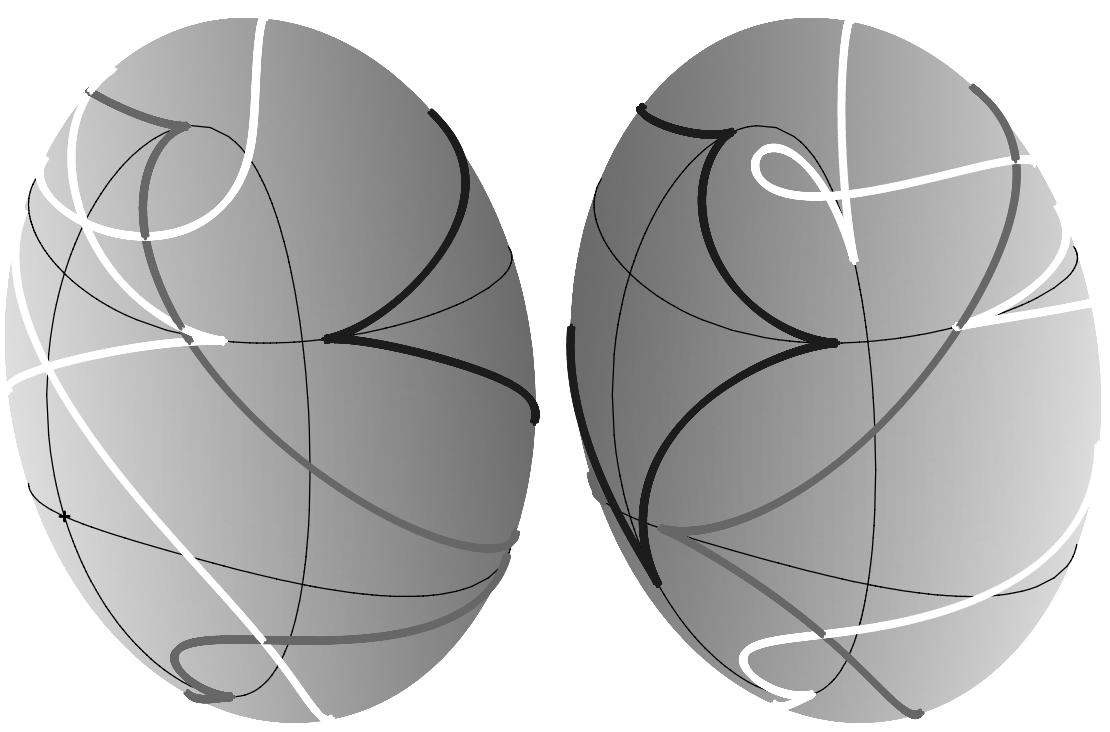

FIGURE 8. The first (black), second (grey), and third (white) caustics from a point on a triaxial ellipsoid. The starting point is indicated by the small black cross on the left view. The thin black lines are lines of curvature.

We can also update the direction vector:

$$
\vec{v}_{1}=\vec{x}_{1}-\vec{x}_{0} .
$$

These equations are iterated a given number of times for the same starting point but two (close) initial directions. We then find intersections of these piecewise-linear approximations to the two close geodesics $\left(x\right.$ and $\left.x^{\prime}\right)$ by computing

$$
\left\{\vec{x}_{i}-\vec{x}_{i}^{\prime}\right\} \cdot \nabla U\left(\vec{x}_{i}\right) \times \vec{v}_{i}
$$

and looking for its zeros as one does for a Jacobi field, which this simple algorithm is designed to mimic.

\subsection{Two Examples of Triaxial Ellipsoids}

For the first example, we make use of a modified version of the software package Loki [Sinclair and Tanaka 02].
This version can compute cut loci from a restricted set of starting points on triaxial ellipsoids.

We use the surface given by

$$
\frac{25 x^{2}}{64}+\frac{100 y^{2}}{529}+\frac{z^{2}}{4}=1
$$

with the starting point

$$
p_{1}=(0.8644836896,0,1.682941970) .
$$

Figure 7 shows the conjugate locus, the cut locus, and the various lines of curvature intersecting $p_{1}$ or $-p_{1}$. Note that the cut locus is a subarc of a line of curvature, as conjectured in [Itoh and Sinclair 02] (this is an independent check of the results of that paper because here we are using entirely different software to compute the cut locus), and that the endpoints of the cut locus are cusps of the conjugate locus. 


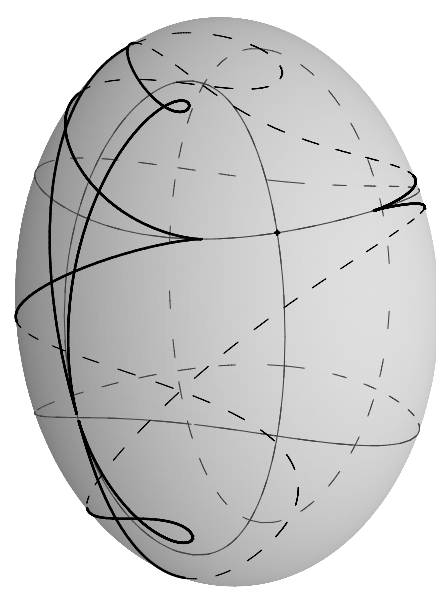

FIGURE 9. The fourth caustic from a point on a triaxial ellipsoid. The starting point is indicated by a small black cross. The thin dark lines are lines of curvature.

For the second example, we use the surface given by

$$
\frac{25 x^{2}}{64}+\frac{100 y^{2}}{529}+\frac{z^{2}}{9}=1
$$

with the starting point

$$
p_{2}=(1.268661,1.131879,1.077939) .
$$

In Figure 8, we can see the first, second, and third caustics. The fourth caustic is in Figure 9. What is striking is the role played by the lines of curvature passing through $p_{2}$ or $-p_{2}$. The cusps of the caustics meet these lines tangentially (as von Braunmühl showed for the first caustic), but not all of the self-intersections of the third and fourth caustics are on one of these lines of curvature.

In all cases, the caustics have four cusps.

\section{CONCLUSION}

Our results appear to give one good reason for believing that the statement

all caustics from non-umbilical points $p$ on triaxial ellipsoids (or non-polar points $p$ on ellipsoids of revolution) have exactly four cusps, which are to be found on the lines of curvature (or meridians and parallels) intersecting $p$ (for even caustics) or $-p$ (for odd caustics), meeting these lines tangentially

is true, where an $n^{\text {th }}$ caustic is called even if $n$ is even, and odd if $n$ is odd. Whatever he might actually have written, we feel that it remains appropriate to attribute this claim to Jacobi.

\section{ACKNOWLEDGMENTS}

The author would like to thank Prof. M. Berger of IHÉS, France, for constructive criticism of a draft of the paper that has resulted in some much needed improvements. He would also like to thank Prof. H. Knörrer of the ETH, Switzerland, for also checking a draft, and Profs. M. Tanaka of Tokai University, Japan and H. Rubinstein of The University of Melbourne for many useful discussions.

\section{REFERENCES}

[Arnold 94] V. I. Arnold. Topological Invariants of Plane Curves and Caustics, University Lecture Series, Vol. 5. Providence, RI: AMS, 1994.

[Arnold 99] V. I. Arnold. "Topological Problems in Wave Propagation Theory and Topological Economy Principle in Algebraic Geometry." In The Arnoldfest, edited by Edward Bierstone et al., pp. 39-54. Fields Institute Communications, Vol. 24. Providence, RI: AMS, 1999.

[Benamou and Solliec 00] J. -D. Benamou and I. Solliec. "A Eulerian Method for Capturing Caustics." Journal of Computational Physics 162:1 (2000), 132-163.

[Berger 00] M. Berger. Riemannian Geometry During the Second Half of the Twentieth Century, University Lecture Series, Vol. 17. Providence, RI: AMS, 2000.

[Bolsinov and Fomenko 99] A. V. Bolsinov and A. T. Fomenko. Integrable Geodesic Flows on TwoDimensional Surfaces, Monographs in Contemporary Mathematics. New York: Kluwer Academic/Plenum Publishers, 1999.

[von Braunmühl 79] A. von Braunmühl. "Ueber Enveloppen geodätischer Linien." Mathematische Annalen XIV (1879), 557-566 with one lithographic print.

[von Braunmühl 82] A. von Braunmühl. "Geodätische Linien und ihre Enveloppen auf dreiaxigen Flächen zweiten Grades." Mathematische Annalen XX (1882), 557-586 with one lithographic print.

[do Carmo 92] M. P. do Carmo. Riemannian Geometry. Boston: Birkhäuser, 1992.

[Duistermaat 74] J. J. Duistermaat. "Oscillatory Integrals, Lagrange Immersions and Unfolding of Singularities." Communications on Pure and Applied Mathematics XXVII (1974), 207-281.

[Ehlers and Newman 00] J. Ehlers and E. T. Newman. "The Theory of Caustics and Wave Front Singularities with Physical Applications." Journal of Mathematical Physics 41:6 (2000), 3344-3378.

[Engquist et al. 02] B. Engquist, O. Runborg, and A. -K. Tornberg. "High-Frequency Wave Propagation by the Segment Projection Method." Journal of Computational Physics 178:2 (2002), 373-390.

[Featherstone 96] W. E. Featherstone. "A Compendium of Earth Constants Relevant to Australian Geodetic Science." Geomatics Research Australasia 64 (1996), 65-74. 
[Fomel and Sethian 02] S. Fomel and J. A. Sethian. "FastPhase Space Computation of Multiple Arrivals." Proceedings of the National Academy of Sciences of the USA 99:11 (2002), 7329-7334.

[Giaquinta and Hildebrandt 96] M. Giaquinta and S. Hildebrandt. Calculus of Variations I, Grundlehren der mathematischen Wissenschaften 310. Berlin: Springer-Verlag, 1996.

[Gjøystdal et al. 02] H. Gjøystdal, E. Iversen, R. Laurain, I. Lecomte, V. Vinje, and K. Åstebøl. "Review of Ray Theory Applications in Modelling and Imaging of Seismic Data." Studia Geophysica et Geodaetica 46:2 (2002), 113164.

[Hanyga 97] A. Hanyga. "Canonical Functions of Asymptotic Diffraction Theory Associated with Symplectic Singularities." In Symplectic Singularities and Geometry of Gauge Fields, Banach Center Publications, Vol. 39, pp. 57-71. Warszawa: Institute of Mathematics, Polish Academy of Sciences, 1997.

[Itoh and Sinclair 02] J. -I. Itoh and R. Sinclair. "Thaw: A Tool for Approximating Cut Loci on a Triangulation of a Surface." Submitted to Experimental Mathematics, 2002.

[Joets and Ribotta 99] A. Joets and R. Ribotta. "Caustique de la surface ellipsoïdale à trois dimensions." Experimental Mathematics 8:1 (1999), 49-55.

[Jacobi 84] C. G .J. Jacobi. "Vorlesungen über Dynamik." In C.G.J. Jacobi's Gesammelte Werke, Second edition, Supplement Volume, edited by A. Clebsch and E. Lottner, pp. 46-47. Berlin: Georg Reimer, 1884.

[Jacobi and Wangerin 91] C. G. J. Jacobi and A. Wangerin. "Über die Kurve, welche alle von einem Punkte ausgehenden geodätischen Linien eines Rotationsellipsoides berührt." In C. G. J. Jacobi's Gesammelte Werke, Volume 7, edited by K. Weierstrass, pp. 72-87. Berlin: Georg Reimer, 1891.

[Klingenberg 82] W. Klingenberg. Riemannian Geometry. Berlin: Walter de Gruyter, 1982.

[Knörrer 80] H. Knörrer. "Geodesics on the Ellipsoid." Inventiones Mathematicae 59 (1980), 119-143.
[Kobayashi 67] S. Kobayashi. "On Conjugate and Cut Loci." In Studies in Global Geometry and Analysis, Studies in Mathematics, Volume 4, edited by S.S. Chern, pp. 96122. Providence, RI: AMS, 1967.

[Lambare et al. 96] G. Lambare, P. S. Lucio, and A. Hanyga. "Two-Dimensional Multivalued Traveltime and Amplitude Maps by Uniform Sampling of a Ray Field." Geophysical Journal International 125:2 (1996), 584-598.

[Longuet-Higgins 90] M. Longuet-Higgins. "Ray Paths and Caustics on a Slightly Oblate Ellipsoid." Proceedings of the Royal Society of London, Series A 428 (1990), 283290.

[von Mangoldt 81] H. von Mangoldt. "Ueber diejenigen Punkte auf positiv gekrümmten Flächen, welche die Eigenschaft haben, dass die von ihnen ausgehenden geodätischen Linien nie aufhören, kürzeste Linien zu sein." Journal für die reine und angewandte Mathematik XCI:1 (1881), 23-53 with one lithographic print (Figure 2 of Print I).

[Margerin 91] C. M. Margerin. "General Conjugate Loci Are Not Closed." In Differential Geometry: Riemannian Geometry, Proceedings of Symposia in Pure Mathematics, Volume 54, Part 3, edited by R. Greene and S. T. Yau, pp. 465-478. Providence, RI: AMS, 1991.

[Poincaré 05] H. Poincaré. "Sur les lignes géodésiques des surfaces convexes." Transactions of the American Mathematical Society 6 (1905), 237-274.

[Postnikov 01] M. M. Postnikov. Geometry VI: Riemannian Geometry, Encyclopaedia of Mathematical Sciences, Vol. 91. Berlin: Springer-Verlag, 2001.

[Sakai 96] T. Sakai. Riemannian Geometry, Translations of Mathematical Monographs, Vol. 149. Providence, RI: AMS, 1996.

[Sinclair and Tanaka 02] R. Sinclair and M. Tanaka. "Loki: Software for Computing Cut Loci." Experimental Mathematics 11:1 (2002), 1-25.

[Struik 61] D. J. Struik. Lectures on Classical Differential Geometry, Second edition. Reading, MA: Addison-Wesley, 1961.

[Wolter 79] F. -E. Wolter. "Distance Function and Cut Loci on a Complete Riemannian Manifold." Archiv der Mathematik 32 (1979), 92-96.

Robert Sinclair, Department of Mathematics and Statistics, The University of Melbourne, Parkville, VIC 3010, Australia (R.Sinclair@ms.unimelb.edu.au)

Received January 23, 2003; accepted in revised form September 23, 2003. 